\title{
Evidências das intervenções na prevenção do trauma mamilar na amamentação: revisão integrativa
}

\author{
Evidence of interventions to avoid nipple trauma in breastfeeding: an integrative review
}

Evidencias de las intervenciones en la prevención del trauma del pezón en la lactancia: revisión integrativa

Andrea de Araújo Costa ${ }^{1}$, Elaine Belem de Souzaㄹ, Janaina Valadares Guimarães ${ }^{3}$, Flaviana Vieira ${ }^{4}$

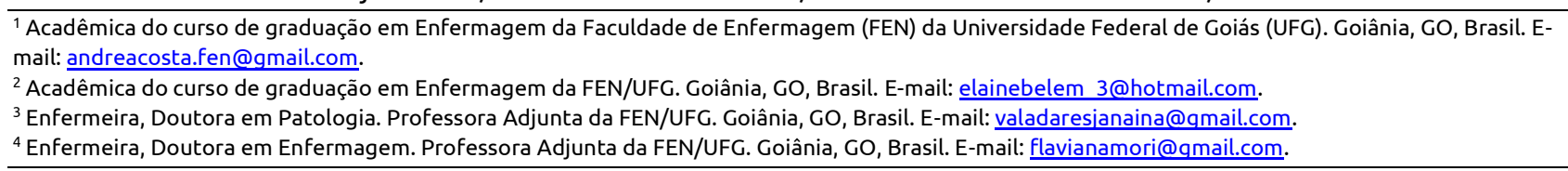

\section{RESUMO}

O trauma mamilar é um dos fatores que contribuem para o desmame precoce, gera dor e desconforto na mãe ao amamentar. O objetivo do estudo foi avaliar as evidências das intervenções para prevenção do trauma mamilar em lactantes. Revisão integrativa realizada na MEDLINE, ScienceDirect, CINAHL, LILACS e SciELO. Foram encontradas oito publicações que atenderam aos critérios de inclusão. Os resultados mostraram três categorias de intervenção: quatro referentes à técnica da amamentação, três por coberturas tópicas e um por outro tipo (presença do companheiro). O gel de menta apresentou forte recomendação para a prevenção do trauma; a técnica correta de amamentação foi controversa; houve contraindicação para o uso de sabão, álcool e pomadas. Considera-se que este estudo contribui para o embasamento da prática clínica dos profissionais e para o direcionamento de pesquisas clínicas futuras, necessárias para as evidências científicas da assistência prestada à lactante na prevenção do trauma mamilar.

Descritores: Aleitamento Materno; Enfermagem Obstétrica; Mamilos; Ferimentos e Lesões.

\section{ABSTRACT}

Nipple trauma is among the factors that contribute with early weaning, and cause pain and discomfort to mothers while breastfeeding. The objective of this study was to evaluate the evidence of the interventions used to avoid nipple trauma in breastfeeding mothers. This integrative review was performed using: MEDLINE, ScienceDirect, CINAHL, LILACS and SciELO. Eight articles that met the inclusion criteria were located. The results revealed three intervention categories: four regarding the breastfeeding technique, three related to ointments and one different type (the presence of the partner). The mint gel was highly recommended to avoid trauma; the correct breastfeeding technique was controversial; there was contradiction for the use of soap, alcohol, and ointments. It is considered that this study helps provide support for the clinical practice and to guide further scientific studies, which are necessary for obtaining scientific evidence of the care provided to the breastfeeding woman to avoid nipple trauma.

Descriptors: Breast Feeding; Obstetrical Nursing; Nipples; Wounds and Injuries.

\section{RESUMEN}

El trauma del pezón es uno de los factores que contribuyen al destete precoz, genera dolor e incomodidad al amamantar. Se objetivó evaluar las evidencias de intervenciones para prevención del trauma del pezón en lactantes. Revisión integrativa realizada en MEDLINE, ScienceDirect, CINAHL, LILACS y SciELO. Fueron encontradas ocho publicaciones atendiendo criterios de inclusión. Los resultados mostraron tres categorías de intervención: cuatro referentes a la técnica de lactancia, tres por coberturas tópicas y una por otro tipo (presencia del compañero). El gel de menta fue altamente recomendado para prevención del trauma; la técnica correcta de amamantamiento fue controversial; hubo contraindicación para uso de jabón, alcohol y pomadas. Se considera que este estudio contribuye a la base de la práctica clínica profesional y para orientar futuras investigaciones clínicas, necesarias para las evidencias científicas de la atención brindada a la lactante en la prevención del trauma del pezón.

Descriptores: Lactancia Materna; Enfermería Obstétrica; Pezones; Heridas y Traumatismos. 


\section{INTRODUÇÃO}

O aleitamento materno (AM) é muito mais do que nutrir uma criança, é a mais sábia estratégia natural de interação profunda entre mãe e filho, afeto, habilidade de se defender de infecções, em sua fisiologia e no seu desenvolvimento cognitivo e emocional, além de ter implicações na saúde física e psíquica da mãe. Constitui a mais sensível, econômica e eficaz intervenção para redução da morbimortalidade infantil(1).

Chega a menos de $40 \%$ a porcentagem de crianças com até seis meses de idade que são amamentadas exclusivamente, revelando a necessidade de intervenção profissional para as mães ${ }^{(2)}$.

A recomendação de Organizações de Saúde nacionais e internacionais, Ministério da Saúde (MS) e o Fundo das Nações Unidas para a Infância (UNICEF), recomendam aleitamento materno exclusivo por seis meses e complementado até os dois anos ou mais. Não há vantagens em se iniciar os alimentos complementares antes dos seis meses, podendo, inclusive, haver prejuízos à saúde da criança ${ }^{(1,3)}$.

A coabitação dos pais, permanência da mãe com a criança em casa nos primeiros seis meses, o não uso de chupeta e a introdução mais tardia (após os seis meses) de outro leite e de chás e/ou água na alimentação da criança são fatores associados à manutenção do aleitamento materno(4).

Porém, há fatores que geralmente contribuem ao desmame precoce, dentre eles a presença de trauma mamilar, a qual é acompanhada pela dor e desconforto da mãe ao amamentar ${ }^{(5)}$.

O trauma mamilar é definido como uma lesão e/ou alteração do tecido mamilar que, geralmente, são resultantes do manejo inadequado e/ou de erro na técnica da amamentação - posicionamento e pega incorreta do lactente ${ }^{(6)}$, podendo aparecer tanto na maternidade quanto nos primeiros sete dias pós parto, variando entre 43,6 a 46,9\%, respectivamente, interferindo no sucesso do $\mathrm{AM}^{(7)}$.

Na maioria das vezes é a má pega da criança ao amamentar que leva ao trauma, que geralmente ocorre no quadrante superior externo do mamilo, porque nesta área incide a força da mandíbula, no ato da sucção(3).

Os fatores como a primiparidade $(57,4 \%)$; o lábio do lactente inferior inadequado (voltado para dentro) (72,9\%) e o queixo do lactente não posicionado de forma adequada (não tocando a mama) (72,2\%) estiveram frequentes nas mulheres com lesão mamilar ${ }^{(6)}$. Porém, os fatores que apresentaram associação significativa $(p<0,05)$ com o trauma foram a ausência do companheiro, o ingurgitamento mamário, a semi-protrusão e/ou a malformação dos mamilos, a despigmentação mamilar e amamentação na primeira hora de vida do lactente ${ }^{(8)}$.

Diante disso, foi observado que estudos sobre amamentação têm abordado mais os fatores associados ao trauma, prevalência de amamentação(4,8), por outro lado, estudos na área de prevenção de trauma mamilar são escassos.

Por isso, o uso da revisão integrativa, com o levantamento das intervenções eficazes para prevenção do trauma mamilar, torna-se de suma importância na prática da enfermagem para subsidiar a assistência à puérpera no processo de amamentação, prolongando possivelmente a duração deste em benefício para o bebê quanto para a mãe.

A utilização da Prática Baseada em Evidência (PBE) incorporará a melhor evidência científica para o uso nas tomadas de decisões na prática clínica e na intervenção individual no paciente ${ }^{(9)}$ como também nas intervenções de caráter preventivo na ocorrência do trauma mamilar. Uma das finalidades da PBE é incentivar a utilização de resultados dessas pesquisas associada à assistência à saúde reforçando a importância da pesquisa para a prática clínica prestada nos vários níveis de atenção(10).

Diante do contexto da necessidade de prevenção do trauma mamilar, a seguinte questão norteou o direcionamento do estudo: "Quais são as intervenções eficazes para prevenção do trauma mamilar em lactantes?"

Procurando contribuir para ampliação do conhecimento de enfermagem com impacto no ensino, pesquisa e na assistência, tem-se como objetivo avaliar as evidências das intervenções para prevenção do trauma mamilar em lactantes.

\section{METODOLOGIA}

Este estudo consiste em uma revisão bibliográfica desenvolvida por meio do método de Revisão Integrativa. Esta se compõe por seis fases que foram utilizadas para o desenvolvimento deste estudo: identificação do tema e seleção da hipótese; amostragem ou busca na literatura; categorização dos estudos; avaliação dos estudos incluídos na revisão; interpretação dos resultados e apresentação da revisão(10). 
A busca dos artigos foi realizada nas bases de dados MEDLINE (Literatura Internacional em Ciências da Saúde), ScienceDirect (Elselvier), CINAHL (Cumulative Index of Nursing and Allied Health Literature) LILACS (Literatura Latino-Americana e do Caribe em Ciências da Saúde) e na biblioteca SciELO (Scientific Electronic Library Online).

Estas bases foram acessadas por meio da Biblioteca Virtual em Saúde Pública (BVS)/Bireme/OMS (http://bases.bvs.br) e Portal de Periódicos CAPES (http://novo.periodicos.capes.gov.br).

Os critérios de inclusão adotados para orientar a busca e seleção dos artigos foram: abordar a temática prevenção do trauma mamilar, apresentar-se nos idiomas português, espanhol e inglês e publicado na íntegra (disponível online ou por comutação). Os critérios de exclusão estabelecidos foram: estudos do tipo qualitativo, publicações do tipo carta editorial, revisão de literatura ou revisão integrativa.

A estratégia de busca foi baseada com os descritores padronizados: mamilos, aleitamento materno e ferimentos e lesões; e não padronizado: prevenção. Os termos padronizados foram identificados no Medical Subject Heading Section (MeSH) e nos Descritores em Ciências da Saúde (DeCS). O período de publicação não foi delimitado para a possibilidade de ampliar o número de publicações.

As estratégias de busca foram: nipples and prevention or (nipples and breast feeding) or (nipples and wounds and injuries) or (breast feeding and wounds and injuries) or (nipples and prevention and breast feeding) or (nipples and breast feeding and wounds and injuries) or (prevention and breast feeding and wounds and injuries) or (nipples and prevention and wounds and injuries) or (nipples and prevention and wounds and injuries and breast feeding).

Na $1^{\text {a }}$ fase, para a seleção dos artigos foi realizada uma leitura criteriosa dos títulos e resumos de todas as publicações localizadas pela estratégia de busca a fim de verificar a adequação aos critérios de inclusão respondendo a todas as opções do formulário (fonte, ano, título, tema, idioma, publicação na íntegra). Nos casos em que o título e o resumo não foram suficientes para definir a sua primeira seleção, estes foram buscados na íntegra. As publicações que não estavam na íntegra na busca virtual foram comutadas para avaliação de inclusão.

Em seguida, na $2^{\text {a }}$ fase, foi realizada a leitura na íntegra de cada estudo pré-selecionado. A avaliação dos estudos foi feita concomitante e independente por dois pesquisadores. O terceiro pesquisador foi acionado quando houve divergência na decisão de inclusão ou não do estudo. Nesta fase os estudos repetidos foram eliminados.

Foi elaborado, no final do estudo, um fluxograma da coleta de dados e seleção dos estudos (Figura 1). Os estudos excluídos foram registrados em porcentagens a partir do motivo da sua exclusão. Para melhor identificação os estudos selecionados receberam um código de sequência alfanumérica $\left(E_{1}, E_{2}, E_{3} \ldots\right)$. 
Figura 1: Seleção dos artigos.

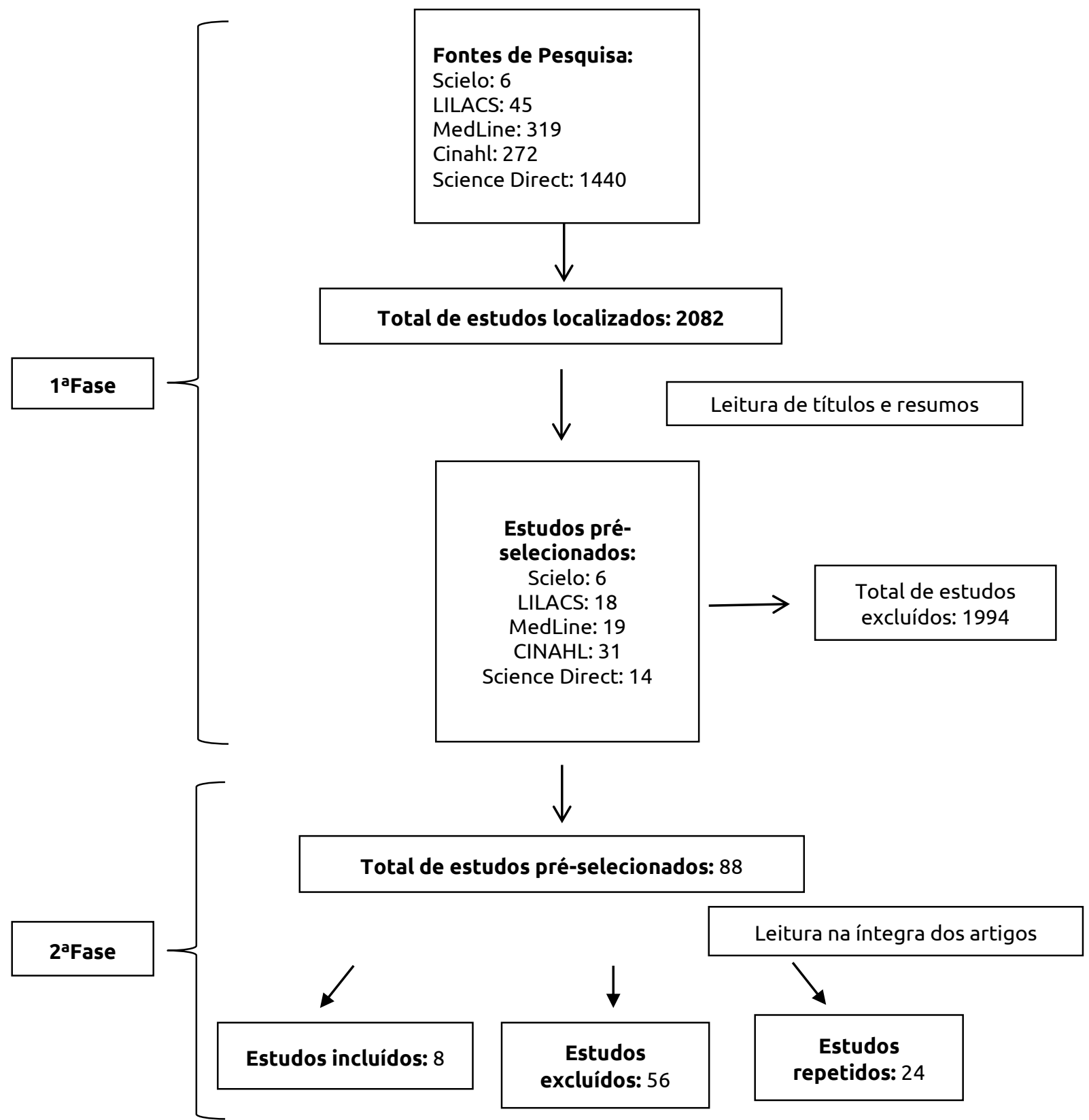

Para extração dos dados foi elaborado um quadro sinótico com a finalidade de categorizar os estudos incluídos contendo os seguintes aspectos: autor/título/ano/país da publicação, metodologia, resultados e níveis de evidência.

Após este passo foi realizado uma leitura criteriosa dos estudos selecionados a fim de identificar possíveis intervenções para prevenção do trauma mamilar e os níveis de evidência do estudo.

A análise foi realizada de forma crítica baseada nos cinco níveis de evidências e nos quatro graus de recomendação de Oxford ${ }^{(11)}$.

Os níveis de evidência são classificados em: 1 Revisão sistemática de ensaios clínicos controlados e randomizados; 2 - Ensaio clínico randomizado ou estudo observacional; 3 - Estudo de coorte com grupo controle não randomizado/estudo de seguimento; 4 - Série de casos, estudos de caso-controle ou estudos historicamente controlados; 5 - Mecanismos baseados em raciocínios (opinião desprovida de avaliação crítica explícita ou baseada em princípios básicos - estudos fisiológicos ou estudos em animais).

Os graus de recomendações: A (estudos de nível 1); B (estudos de nível 2 e 3); C (estudos de nível 4) e D (estudos de nivel 5 ou problematicamente inconsistentes ou inconclusivos). O grau de recomendação A representa alto nível de evidência, $B$ moderado, C e D menor nível de evidência. 
A localização dos textos na íntegra foi possível com o acesso às Bibliotecas Eletrônicas para 95,5\% dos trabalhos na Faculdade de Enfermagem e na Biblioteca Central da Universidade Federal de Goiás. Outros 4,5\% foram comutados.

\section{RESULTADOS}

Entre os 2.082 artigos localizados na busca, 1.140 $(69,16 \%)$ foram encontrados na Science Direct, 319 (15,32\%) na MedLine, 272 (13,06\%) na CINAHL, 45 (2,17\%) na LILACS e $6(0,29 \%)$ na Scielo.

A partir dos estudos encontrados, 1.994 foram excluídos por não abordarem o tema de prevenção do trauma mamilar. Do total de artigos pré-selecionados, 56 foram excluídos: 21 (37,5\%) são estudos de revisões (narrativa e integrativa), 14 (25\%) refere a tratamento do trauma mamilar, $12(21,4 \%)$ não abordaram o tema, 9 $(16,1 \%)$ diz respeito a dor. Dentre os artigos préselecionados 24 eram de repetição.

Dentre os oito artigos incluídos, três são de autoria de enfermeiros e médicos, dois possuem enfermeiro, médico, nutricionista e fonoaudiólogo, em dois não foi possível identificar a categoria profissional de seus autores e um tem somente médicos como autores.

Os artigos estão publicados no Jornal de Pediatria (dois), Journal of Human Lactation (dois), Archivos de Pediatría del Uruguay (um), The Journal of Pediatrics (um), Medical Science Monitor (um) e na Revista Escola de Enfermagem da USP (um).

A leitura dos artigos selecionados permitiu agrupar os resultados em quadros por intervenções estudadas, seguindo ordem do ano de publicação. Constituíram-se três categorias de análise: quatro (50\%) referentes à intervenção por técnica da amamentação, três $(37,5 \%)$ por coberturas tópicas e um $(12,5 \%)$ por outro tipo de intervenção.

\section{Intervenção quanto à técnica da amamentação}

Os quatro artigos que compuseram esta categoria abordaram de forma geral a técnica de amamentação principalmente o posicionamento mãe/bebê e a pega do bebê (Quadro 1). 
Quadro 1: Distribuição dos artigos caracterizados pela intervenção quanto à técnica da amamentação.

\begin{tabular}{|c|c|c|c|c|}
\hline Autor/Título/Ano/ País & Objetivo & Método & Resultado & $\begin{array}{l}\text { Nível de } \\
\text { Evidência }\end{array}$ \\
\hline $\begin{array}{c}\text { E1: Moraes M, Da Silva L, Faliú B, Sosa C. } \\
\text { Técnica de alimentación a pecho y aparición } \\
\text { de trauma del pezón previo al alta } \\
\text { hospitalaria. Ano: 2011. País: Uruguai }\end{array}$ & $\begin{array}{c}\text { Descrever e analisar a técnica da } \\
\text { amamentação e a presença de } \\
\text { trauma mamilar antes da alta } \\
\text { hospitalar. }\end{array}$ & $\begin{array}{l}\text { Estudo de coorte transversal, } \\
\text { prospectivo, com } 204 \text { mães em } \\
\text { alojamento conjunto antes da } \\
\text { alta hospitalar entre } 48 \text { e } 96 \\
\text { horas pós-parto. }\end{array}$ & $\begin{array}{c}\text { Alterações da posição da mãe e bebê ao } \\
\text { amamentar foi estatisticamente significativa } \\
\text { para a aparição de fissuras no mamilo }(p=0,01) \text {. } \\
\text { Ter um ou mais filhos anteriormente reduz a } \\
\text { aparição de fissura mamilar e outras } \\
\text { complicações mamárias }(p=0,01) \text {. }\end{array}$ & Nível 3 \\
\hline $\begin{array}{c}\text { E2: Coca KP, Gamba MA, Silva RS, Abrao ACFV. } \\
\text { A posição de amamentar determina o } \\
\text { aparecimento do trauma mamilar? } \\
\text { Ano: 2009a. } \\
\text { País: Brasil. }\end{array}$ & $\begin{array}{l}\text { Identificar as variáveis } \\
\text { relacionadas ao posicionamento } \\
\text { e apreensão durante a } \\
\text { amamentação e o aparecimento } \\
\text { de traumas mamilares. }\end{array}$ & $\begin{array}{l}\text { Estudo caso-controle com } 146 \\
\text { puérperas internadas em um } \\
\text { hospital Universitário durante a } \\
\text { primeira semana pós-parto. }\end{array}$ & $\begin{array}{c}\text { O acompanhamento do posicionamento do } \\
\text { bebê determinou a prevenção do trauma } \\
\text { mamilar no início da amamentação e a sua } \\
\text { continuidade. } \\
\text { Os parâmetros: posição da criança desalinhada } \\
\text { ( } p=0,047) \text {, queixo distante da mama }(p=0,044) \\
\text { e lábio voltado para dentro }(p=0,001) \text { foram } \\
\text { estatisticamente significativos para o } \\
\text { aparecimento do trauma mamilar. }\end{array}$ & Nível 4 \\
\hline $\begin{array}{c}\text { E3: Oliveira LD, Giugliani ER, Do Espírito Santo } \\
\text { LC, Cavalheiro M, França T, Weigert EML, et al. } \\
\text { Effect of Intervention to Improve } \\
\text { Breastfeeding Technique on the Frequency of } \\
\text { Exclusive Breastfeeding and Lactation- } \\
\text { Related Problems. Ano: } 2006 . \\
\text { País: Brasil. }\end{array}$ & $\begin{array}{l}\text { Avaliar o impacto de uma } \\
\text { intervenção da técnica de } \\
\text { amamentação sobre a taxa de } \\
\text { aleitamento materno exclusivo e } \\
\text { problemas mamários } \\
\text { relacionados a amamentação, } \\
\text { durante o primeiro mês pós- } \\
\text { parto. }\end{array}$ & $\begin{array}{l}\text { Ensaio clínico randomizado com } \\
211 \text { pares (mãe e filho) em uma } \\
\text { maternidade, aos } 7 \text { e } 30 \text { dias após } \\
\text { o parto. } \\
\text { GI (grupo intervenção): ( } n=74) \\
\text { orientação sobre técnica correta } \\
\text { de amamentação mais a rotina da } \\
\text { maternidade. } \\
\text { GC (grupo controle): ( } n=137 \text { ) } \\
\text { recebeu a rotina da maternidade. }\end{array}$ & $\begin{array}{c}\text { Uma única intervenção na maternidade não foi } \\
\text { suficiente para melhorar a técnica de } \\
\text { amamentação, aumentar as taxas de AME nos } \\
7 \text { ( } p=0,760 \text { ) e } 30 \text { dias ( } p=365 \text { ), e reduzir a } \\
\text { incidência de problemas mamários } \\
\text { relacionados à amamentação. } \\
\text { A frequência do trauma mamilar com } 7 \text { dias foi } \\
\text { de } 43,2 \% \text { no GC e } 48,9 \% \text { no Gl. Quase metade } \\
\text { das mães tiveram trauma mamilar } \\
\text { independente de terem recebido ou não a } \\
\text { intervenção. } \\
\text { As intercorrências mamárias (trauma mamilar, } \\
\text { ingurgitamento e mastite) foram similares nos } \\
\text { grupos aos } 7 \text { e } 30 \text { dias de pós-parto. }\end{array}$ & Nível 1 \\
\hline $\begin{array}{l}\text { E4: Weigert EML, Giuglian ERJ, França MCT, } \\
\text { De Oliveira LD, Bonilha A, Do Espírito Santo LC } \\
\text { et al. Influência da técnica de amamentação } \\
\text { nas frequências de aleitamento materno } \\
\text { exclusivo e lesões mamilares no primeiro mês } \\
\text { de lactação. } \\
\text { Ano: 2005. País: Brasil. }\end{array}$ & $\begin{array}{l}\text { Investigar a influência da técnica } \\
\text { de amamentação nas } \\
\text { frequências de AME e na } \\
\text { ocorrência de lesões mamilares } \\
\text { no primeiro mês de lactação. }\end{array}$ & $\begin{array}{l}\text { Estudo de coorte } \\
\text { contemporâneo, observacional, } \\
\text { com } 211 \text { mães/bebê na } \\
\text { maternidade, aos } 7 \text { e aos } 30 \text { dias } \\
\text { após o parto. }\end{array}$ & $\begin{array}{l}\text { A técnica de amamentação não influenciou na } \\
\text { redução da ocorrência do trauma mamilar. } \\
\text { As mulheres com trauma mamilar } \\
\text { apresentaram em média menos parâmetros } \\
\text { desfavoráveis (posicionamento mãe/bebê) do } \\
\text { que as que não tiveram trauma( } p=0,04) \text {. } \\
\text { No parâmetro desfavorável relativos à pega } \\
\text { não houve diferença significativa }(p=0,189) \text {. }\end{array}$ & Nível 3 \\
\hline
\end{tabular}


Houve uma relação positiva entre o posicionamento e a pega adequados para a prevenção do trauma mamilar nos estudos E1 e E2, tendo respectivamente, evidência moderada (B) e fraca $(C)^{(6,12)}$. Porém, no estudo E3 e E4 não foi encontrada relação(13-14).

Pode-se dizer que a presença da posição adequada do bebê relacionado à mãe e a pega correta pelo bebê previnem o trauma mamilar. Uma vez que, as lactantes que durante a mamada seus bebês apresentaram: posição desalinhada $(p=0,047)$, pescoço torcido, queixo distante da mama $(p=0,044)$ e o lábio inferior voltado para dentro $(p=0,001)$, tem mais trauma mamilar quando comparadas as lactantes sem estas características ${ }^{(6)}$.

O estudo E3, apesar de a intervenção ter sido realizada por duas enfermeiras com vasta experiência no aconselhamento em amamentação e certificando-se de que todos os tópicos importantes foram abordados, mostrou que uma única intervenção na maternidade possivelmente não foi suficiente para melhorar a técnica de amamentação e reduzir a incidência de problemas mamários, ressaltando que poderia ser mais eficaz se houvesse mais de um contato com as mães ${ }^{(13)}(A)$.

No estudo E4, a técnica de amamentação não influenciou na redução da ocorrência do trauma mamilar (B). Das puérperas que foram pesquisadas na alta hospitalar quanto a técnica de amamentação, 52,2\%, das que apresentaram lesões mamilares, e $38,7 \%$, das que não apresentaram tais lesões, tinham sido assistidas pela equipe especializada no manejo do AM. Esta equipe atende preferencialmente as puérperas que apresentam dificuldades na amamentação(14).

\section{Intervenção por coberturas tópicas}

Os tipos de coberturas estudados foram o gel de menta, lanolina, vitamina A e D, água e sabão e álcool a $70 \%$. Somente uma publicação é da última década (Quadro 2). 
Quadro 2: Intervenção por coberturas tópicas.

\begin{tabular}{|c|c|c|c|c|}
\hline Autor/Título/Ano/ País & Objetivo & Método & Resultado & $\begin{array}{c}\text { Nível de } \\
\text { Evidência }\end{array}$ \\
\hline $\begin{array}{c}\text { E5: Melli MS, Rashidi MR, Nokhoodchi A, } \\
\text { Tagavi S, Farzadi L, Sadaghat K, } \\
\text { Tahmasebi Z, Sheshvan MK. A } \\
\text { randomized trial of peppermint gel, } \\
\text { lanolina ointment, and placebo gel to } \\
\text { prevent nipple crack in primiparous } \\
\text { breastfeeding women. } \\
\text { Ano: } 2007 . \\
\text { País: Iran. }\end{array}$ & $\begin{array}{c}\text { Investigar o efeito } \\
\text { preventivo do gel de } \\
\text { menta para fissura no } \\
\text { mamilo em primíparas em } \\
\text { lactação. }\end{array}$ & $\begin{array}{l}\text { Estudo duplo-cego, randomizado, } \\
\text { constituído por } 206 \text { primíparas, divididas } \\
\text { em três grupos: lanolina pura, gel de menta } \\
\text { e gel placebo. } \\
\text { As participantes foram acompanhadas por } \\
\text { entrevista telefônica realizadas nos dias } 4 \text {, } \\
7,10,14 \text {, e } 42 \text { pós-parto. }\end{array}$ & $\begin{array}{c}\text { As diferenças médias entre os grupos com relação a } \\
\text { gravidade de fissura do mamilo foram significativo ( } p \\
=0,004 \text { ). O grupo do gel de menta teve taxa mínima } \\
\text { de } 3,8 \% \text { de fissura e o grupo do gel placebo } \\
\text { apresentou taxa máxima de } 22,6 \% \text {. A lanolina teve } \\
\text { taxa intermediária de } 6,9 \% \text {. } \\
\text { Mães que receberam gel de menta tiveram menos } \\
\text { fissuras do que aquelas que receberam pomada de } \\
\text { lanolina ou placebo ( } p=0,01 \text { ). }\end{array}$ & Nível 1 \\
\hline $\begin{array}{c}\text { E6: Centuori S, Burmaz T, Ronfani L, } \\
\text { Fragiacomo M, et al. Nipple care, Sore } \\
\text { nipples, and breastfeeding: A } \\
\text { Randomized Trial. } \\
\text { Ano: } 1999 . \\
\text { País: Itália. }\end{array}$ & $\begin{array}{l}\text { Determinar se a } \\
\text { incidência de dor, trauma } \\
\text { mamilar e a duração do } \\
\text { aleitamento materno são } \\
\text { afetados pelo uso de } \\
\text { pomada e creme no } \\
\text { mamilo. }\end{array}$ & $\begin{array}{l}\text { Ensaio clinico randomizado com } 219 \text { mães. } \\
\text { O grupo controle (GC) ( } \mathrm{n}=96 \text { ) seguiu a } \\
\text { rotina do hospital: limpar as mamas com } \\
\text { água estéril antes e após cada mamada, em } \\
\text { caso de dor usar pomada (preparada pela } \\
\text { farmácia do hospital) ou spray comercial, } \\
\text { aplicado após cada mamada e removido } \\
\text { antes da próxima. } \\
\text { O grupo de intervenção (GI) ( } \mathrm{n}=123 \text { ) evitou } \\
\text { o uso de pomada e outros produtos sendo } \\
\text { realizada somente a higienização das } \\
\text { mamas e mamilos nos banhos diários. }\end{array}$ & $\begin{array}{c}\text { Não houve diferença significativa }(p>0,05) \text { entre os } \\
\text { grupos na incidência de trauma mamilar, nem na dor e } \\
\text { nem na duração da amamentação. O uso de pomadas } \\
\text { nos mamilos não influenciou na incidência de dor ou } \\
\text { de trauma ou de amamentação. } \\
\text { A limpeza especial, aplicação de pomadas ou spray nos } \\
\text { mamilos são dispensáveis, sendo mais caros e podem } \\
\text { requerer intervenções desnecessárias. O uso da } \\
\text { chupeta ( } p=0,02) \text {, da mamadeira }(p=0,03) \text { e } \\
\text { suplementação alimentar }(p<0,05) \text { no hospital foram } \\
\text { associados à alta incidência de dor e traumas } \\
\text { mamilares. }\end{array}$ & Nível 1 \\
\hline $\begin{array}{c}\text { E7: Newton, } \mathrm{N} \text {. } \\
\text { Nipple pain and nipple damage: } \\
\text { Problems in the management of breast } \\
\text { feeding. } \\
\text { Ano: } 1952 . \\
\text { País: Estados Unidos. }\end{array}$ & $\begin{array}{l}\text { Estudar a relação de } \\
\text { determinados fatores } \\
\text { físicos e mentais voltado } \\
\text { ao cuidado com o mamilo. }\end{array}$ & $\begin{array}{l}\text { Estudo com grupo experimental, com } 287 \\
\text { mães das enfermarias do alojamento } \\
\text { conjunto de um Hospital até o quinto dia } \\
\text { pós-parto. G1: lavou as mamas com } \\
\text { solução de sabão e água antes das } \\
\text { mamadas; G2: limpou os mamilos com água } \\
\text { e álcool a 70\% antes das mamadas; G3: } \\
\text { grupo controle (GC) usou apenas água; } \\
\text { G4: pomada de vitamina A e D; G5: } \\
\text { concentrado de vitamina A e D G6: lanolina. } \\
\text { G3, G4, G5 e G6: lavava os mamilos com } \\
\text { água antes das mamadas. }\end{array}$ & $\begin{array}{c}\text { Evitar o uso do sabão na higienização dos mamilos, } \\
\text { eliminar tentativas de esterilizar os mamilos (uso do } \\
\text { álcool) e amamentar por livre demanda são ações que } \\
\text { evitam a dor e o trauma mamilar. Não ser primípara } \\
\text { também favorece o não aparecimento de trauma. } \\
\text { trauma mamilar foi significativamente mais } \\
\text { encontrado em mães que usaram álcool }(G 2)(p=0,02) \\
\text { e sabão (G1) ( } p=0,02 \text { ) em comparação } \\
\text { com o GC (G3). Foi maior, também, nas mães em que } \\
\text { os bebês mamaram acima de } 6 \text { vezes nas últimas } 24 \\
\text { horas. Concentrado de vitamina A e D foram irritantes } \\
\text { para a pele dos mamilos de algumas mães e } 60 \% \\
\text { sentiram dor extrema comparada ao GC ( } p=0,02) \text {. } \\
\text { Mães que usaram pomada de vitamina A e } D \text { e lanolina } \\
\text { não tiveram proteção contra a dor e ao trauma } \\
\text { quando comparadas ao GC. }\end{array}$ & Nível 2 \\
\hline
\end{tabular}


$O$ estudo $E 5$, evidenciou que o gel de menta foi superior a lanolina pura e ao gel placebo na prevenção da fissura mamilar, apresentando taxas de trauma de 3,8\%, $6,9 \%$ e $22,6 \%$, respectivamente. O risco relativo de trauma no mamilo no grupo lanolina (RR: 2,41, IC 95\%: 1,20-3,01) foi maior do que no grupo do gel de menta (RR: 1,85, IC 95\%: 1,64-3,10)(15) . Este estudo foi nível de evidência $1 \mathrm{com}$ forte recomendação para o uso do gel de menta como prevenção da fissura mamilar (A).

No estudo E6 não foi encontrada significância estatística $(p>0,05)$ com relação à prevenção do trauma nos dois grupos. Ressaltando que neste estudo, de nível 1 , há forte recomendação para que a limpeza especial (com água estéril) antes e após as mamadas, aplicação de pomadas ou spray nos mamilos não sejam utilizadas com o intuito de prevenção do trauma mamilar ${ }^{(16)}(A)$.

O trauma mamilar esteve frequente em $26 \%$ no grupo controle, que fez uso de pomada nas mamas, e em $19 \%$ no grupo de intervenção, que usou apenas a higienização das mamas nos banhos, não houve diferença significativa entre os grupos $(p<0,05)^{(16)}$.
O estudo E7 aponta que se deve evitar o uso do sabão na higienização dos mamilos e eliminar tentativas de esterilizar os mamilos com o uso do álcool. Portanto, percebe-se que se deve realizar apenas uma higienização simples com água na limpeza dos mamilos durante a amamentação. Não deve lavar os mamilos com sabão, devem ser lavados unicamente uma vez ao dia(17).

A lanolina, pomada de vitamina $A$ e $D$ não tiveram eficácia para a prevenção do aparecimento do trauma ${ }^{(17)}$. $O$ concentrado de vitamina $A$ e $D$, ao invés de promover a proteção para o trauma mamilar, foram irritantes para a pele dos mamilos de algumas mães, além de provocar dor em $60 \%$ delas $^{(17)}$.

\section{Outras intervenções}

Ao avaliarem os fatores associados ao trauma mamilar em mulheres em amamentação na maternidade, encontraram associação significativa da presença do companheiro como fator de proteção para o aparecimento do trauma mamilar(8) (Quadro 3).

Quadro 3: Outras intervenções

\begin{tabular}{|c|c|c|c|c|}
\hline Autor/Título/Ano/ País & Objetivo & Método & Resultado & $\begin{array}{c}\text { Nível de } \\
\text { Evidência }\end{array}$ \\
\hline E8: Coca KP, Gamba MA, & Identificar os fatores & $\begin{array}{c}\text { Estudo caso- } \\
\text { controle com 146 }\end{array}$ & $\begin{array}{c}\text { A presença do } \\
\text { companheiro foi um fator } \\
\text { de proteção ao }\end{array}$ & Nível 4 \\
Fatores associados ao & associados ao trauma & puérperas \\
trauma mamilar na & mamilar em mulheres & internadas nas & desenvolvimento de \\
maternidade. & em aleitamento & enfermarias de & trauma nos mamilos ( $p=$ & \\
Ano: 2009. & materno exclusivo na & alojamento & $0,031)$. & \\
País: Brasil. & maternidade. & conjunto. & & \\
\hline
\end{tabular}

Ao contrário da presença do companheiro, que protegeu do trauma mamilar, outros fatores levaram ao aparecimento do trauma mamilar: estimular a amamentação na primeira hora após o nascimento $(p=0,007)$, mama túrgida e/ou ingurgitamento $(p=0,007)$, mamilos semi-protrusos e/ou malformados $(p=0,013)$ e despigmentação dos mamilos $(p=0,001)^{(8)}$.

\section{DISCUSSÃO}

Na intervenção quanto à técnica da amamentação os parâmetros favoráveis à técnica adequada são, lábio inferior voltado para baixo, queixo tocando a mama, boca bem aberta, aréola mais visível acima do que abaixo da boca do bebê, cabeça alinhada com o corpo, face voltada para o mamilo(3).
No entanto, a intervenção de orientação de técnica correta da amamentação pode ser ineficaz se for realizada uma única vez, sem a continuidade da assistência com o acompanhamento da amamentação.

Em relação ao atendimento da equipe especializada no manejo do AM, este explicaria o porquê das mulheres com trauma mamilar apresentarem em média menos parâmetros desfavoráveis (posicionamento mãe/bebê) do que as que não tiveram trauma $(p=0,04)$, pois estes parâmetros já haviam sido adequados antes da alta hospitalar pela equipe especializada. Os autores consideraram que para identificar a influência da técnica no aparecimento do trauma mamilar o que deve ser avaliado é a primeira mamada, pois na alta hospitalar a técnica recebeu outras interferências ${ }^{(14)}$. 
Torna-se necessário que as orientações quanto à técnica adequada da amamentação sejam iniciadas na gestação durante consultas de enfermagem, de preferência no último trimestre da gestação, pois estas irão direcionar a mãe durante o período de amamentação, tendo como objetivo a prevenção do trauma e continuidade da amamentação(18). Sendo a assistência do enfermeiro continuada no pós-parto domiciliar, respeitando e considerando todo o contexto familiar e cultural que envolve a puérpera, suas experiências, expectativas, desejo em amamentar ou não e seus conhecimento/crenças em relação à amamentação(19).

Os estudos apresentaram níveis de evidência variados, sendo somente um de nível 1 (E3) com forte recomendação $(A)$ de que a uma orientação da técnica de amamentação não reduz o trauma mamilar. Os estudos de nível 3 (E1 e E3) foram controversos sobre a influência da técnica de amamentação na prevenção do trauma mamilar. O estudo de nível 4 foi favorável a indicação da técnica de amamentação (E2), porém, com fraca recomendação (C).

Na intervenção por cobertura tópica, o gel de menta que é um preparo tópico, formulado a partir da menta, a qual é um remédio caseiro utilizado pelas pessoas no Azerbaijão, Província do Irã, para evitar fissura do mamilo. Neste estudo, foram preparadas, por dispersão, várias formulações da composição do gel, variando na concentração dos componentes. As mães deste grupo foram instruídas a passar o preparo sobre os mamilos e aréola após a mamada e lavar antes da próxima ${ }^{(15)}$.

O preparo desta pomada foi feita pela própria farmácia do hospital para ser aplicada depois de cada mamada e removida antes da próxima. Sua composição baseia-se em: albumina, glicerina, óleo de parafina, caseína, lanolina, geleia de petróleo, óxido de zinco, hidrato de sódio e potássio e água destilada. Caso a pomada não estivesse disponível era prescrito o uso spray comercial (acetato de clostebol 0,15 g e sulfato de neomicina $0,15 \mathrm{~g}$ em $30 \mathrm{ml}$ ). Este spray tem na sua composição componentes cicatrizantes a base de esteroide e antibiótico(16).

Pomadas que necessitam de remoção deveriam ser contraindicadas durante a amamentação. Uma vez que a pele da região areolomamilar sofre frequentes fricções pela sucção do bebê nas mamadas, com a necessidade de remoção da pomada antes da mamada gera outra fricção,
Quanto à higienização dos mamilos é necessário limpar as mamas somente com água. Soluções como sabão, álcool, loções, óleos e vaselina devem ser evitados, pois interferem na lubrificação natural da pele, sendo suficiente apenas limpar a mama uma vez ao dia durante a higiene corporal geral. Não é recomendado lavar a mama antes das mamadas, isso retira os óleos protetores além de alterar o cheiro que o bebê pode sentir na mama de sua mãe $\mathrm{e}^{(3)}$.

Em relação ao uso da lanolina pôde ser percebido que esta vem sendo utilizada mais para tratamento do que para prevenção de fissuras mamilares ${ }^{(20)}$.

Já os cremes a base de vitamina $A$ e $D$, podem ser usados sem serem removidos antes das mamadas, não sendo tóxico para o bebê. Embora não haja estudos que comprovem a sua eficácia, existe a prática clínica do uso de cremes à base de vitamina $A$ e $D$, cremes ou pomadas com corticoide, este último para as fissuras mais graves, desde que afastada infecção por fungos ou bactérias ${ }^{(21)}$.

Em síntese, este estudo de nível 2, pode ser considerado com moderada indicação de que a higienização das mamas com sabão ou álcool, o uso de pomada de vitamina $A$ e $D$ e a lanolina não previnem o trauma mamilar (B). Assim, como o concentrado de vitamina $A$ e $D$ são prejudiciais à pele do mamilo (B).

No entanto, vale ressaltar que este estudo é antigo, com uma metodologia complexa, em que muitos grupos foram comparados na mesma pesquisa. Outros estudos clínicos recentes são necessários para estas e novas coberturas, e novas tecnologias de prevenção do trauma mamilar sejam melhor investigadas.

Não há na literatura outros estudos que discutam a presença do companheiro como fator preventivo no aparecimento do trauma. Porém, há estudos que trazem o fator companheiro como eficaz no início e manutenção da amamentação ${ }^{(22-24)}$ e outro mostrou que a ausência de coabitação com o companheiro/pai da criança associou positivamente com a manutenção do $\mathrm{AM}^{(4)}$.

Assim, a presença paterna, no apoio a mãe durante a amamentação mostra-se de grande influência no início e na continuidade do $A M$, cabendo a toda equipe multiprofissional de saúde, incentivar o pai a participar efetivamente neste período ${ }^{(23)}$.

Embora a presença do companheiro tenha sido apontado como fator de proteção para o trauma mamilar, neste estudo de nível de evidência 4 , sua indicação tem o que pode facilitar o aparecimento do trauma mamilar. 
recomendação fraca para o uso na prática clínica da prevenção do trauma mamilar (C).

As publicações selecionadas tiveram participação dos enfermeiros, o que se percebe é que os enfermeiros pesquisadores estão preocupados com a prevenção do trauma mamilar, embora de uma forma incipiente nos estudos clínicos que embasem a prática assistencial.

\section{CONCLUSÃO}

$\mathrm{Na}$ busca das evidências de intervenção para a prevenção do trauma mamilar em lactantes, pode-se dizer que a intervenção com forte grau de recomendação foi a utilização do gel a base de menta.

A técnica correta de amamentação foi controversa na prevenção do trauma mamilar, sendo recomendação forte de que uma única intervenção não foi suficiente para melhorar a técnica ou reduzir os traumas mamilares.

As intervenções que tiveram recomendação forte de contraindicação foram: a limpeza especial dos mamilos com agua estéril antes e após as mamadas; a aplicação de pomada (preparada pelo hospital) após cada mamada e sua remoção antes da próxima mamada e a aplicação do spray comercial, cicatrizante a base de esteroide e antibiótico.

A presença do companheiro, embora tenha sido de fraca recomendação, apresentou-se como fator de proteção para o aparecimento do trauma mamilar.

\section{REFERÊNCIAS}

1. Brasil. Ministério da Saúde. Secretaria de Atenção à Saúde. Departamento de Atenção Básica. Saúde da criança: nutrição infantil: aleitamento materno e alimentação complementar. Série A. Normas e Manuais Técnicos. Cadernos de Atenção Básica - n. 23. [Internet]. Brasília: Ministério da Saúde. 2009 [cited 2012 jul 26] p. 11-2. Available from:

http://bvsms.saude.gov.br/bvs/publicacoes/saude_crianca nutri cao aleitamento alimentacao.pdf.

2. WORLD HEALTH ORGANIZATION (WHO). 10 facts on breastfeeding. World Health Organization. [Internet]. 2012 [cited 2012 jul 28]. Available from:

http://www.who.int/features/factfiles/breastfeeding/facts/en/i ndex.html.

3. UNICEF. Fundo das Nações Unidas para a Infância. Iniciativa Hospital Amigo da Criança: módulo 3: Promovendo e incentivando a amamentação em um Hospital Amigo da Criança: curso de 20 horas para equipes de maternidade /

UNICEF/OMS.[Intenet] Brasília: Ministério da Saúde. 2009 [cited 2012 jul 15]. 276 p. Available from:

http://bvsms.saude.gov.br/bvs/publicacoes/iniciativa hospital amigo crianca modulo3.pdf

4. Martins EJ, Giugliani ERJ. Quem são as mulheres que amamentam por 2 anos ou mais?. J. Pediatr. (Rio J.) [Internet]. 2012 [cited 2012 jul 15]; 88(1):63-73. Available from: http://www.scielo.br/pdf/jped/v88n1/a11v88n01.pdf
Este estudo contribui para o embasamento da prática clínica dos profissionais envolvidos com a amamentação e para o direcionamento de pesquisas clínicas futuras.

Percebe-se uma escassez de estudos que abordem especificamente sobre intervenções de prevenção do trauma mamilar, sendo desenvolvidos predominantemente no âmbito internacional, o que mostra a necessidade imprescindível de pesquisas nacionais que desenvolvam essa temática.

Há, também, a necessidade de ensaios clínicos randomizados que estudem a técnica de amamentação na prevenção do trauma mamilar com seguimento da intervenção e pesquisas sobre outros produtos ou coberturas inovadoras.

O foco no desenvolvimento da produção científica com fortes evidências na prevenção do trauma mamilar poderá embasar a prática clínica de enfermagem prestada à lactante.

Cabe ressaltar o fato de que o enfermeiro pode e deve abraçar a responsabilidade técnica de orientação e acompanhamento sobre amamentação no pré-natal, no pós-parto hospitalar e domiciliar, incluindo ações que incentivem a técnica e o processo de amamentação, apoiando as mulheres para esta prática, além de inserir a figura paterna, a fim de evitar o desmame precoce e diminuir o surgimento de possíveis complicações com a mulher e/ou bebê.

5. Vieira GO, Martins CC, Vieira TO, Oliveira NF, Silva LR. Fatores preditivos da interrupção do aleitamento materno exclusivo no primeiro mês de lactação. J. Pediatr (Rio J.) [Internet]. 2010 [cited 2012 jul 15];86(5):441-444. Available from: http://www.scielo.br/pdf/jped/v86n5/v86n5a15.pdf 6. Coca KP, Gamba MA, Silva RS, Abrao ACFV. A posição de amamentar determina o aparecimento do trauma mamilar?. Rev. esc. enferm. USP [Internet]. 2009a [cited 2012 jul 28];43(2):446-452. Available from:

http://www.scielo.br/pdf/reeusp/v43n2/a26v43n2.pdf

7. França MCT, Giugliani ERJ, Oliveira LD, Weigert EML, Espirito Santo LC, Köhler CV et al. Uso de mamadeira no primeiro mês de vida: determinantes e influência na técnica de amamentação. Rev. Saúde Pública (São Paulo) [Internet]. 2008 [cited 2012 jul 10];42(4):607-614. Available from: http://www.scielo.br/pdf/rsp/v42n4/6206.pdf

8. Coca KP, Gamba MA, Silva RS, Abrão ACFV. Fatores associados ao trauma mamilar na maternidade. J. Pediatr. (Rio

J.) [Internet]. 2009b [cited 2012 jul 15];85(4):341-345.

Available from:

http://www.scielo.br/pdf/jped/v85n4/v85n4a12.pdf

9. Silva AA, Pratica Clinica Baseada em Evidencias na Área de Saúde. 1st ed. São Paulo: Santos; 2009.

10. Mendes KDS, Silveira RCCP, Galvão CM. Revisão integrativa: método de pesquisa para a incorporação de evidências na saúde e na enfermagem. Texto contexto - enferm. [Internet]. 2008 
[cited 2012 jul 28];17(4):758-764. Available

from: http://www.scielo.br/pdf/tce/v17n4/18.pdf.

11. Howick J, Chalmers I, Glasziou P, Greenhalgh T, Heneghan $C$, Liberati A, Moschetti I, Phillips B, Thornton H, Goddard O, Hodgkinson. Oxford Centre for Evidence-based Medicine Levels of Evidence. Evidence-based Medicine Levels of Evidence. Developed byUniversity of OXFORD. [Internet]. 2011 [cited 2012 jul 28]. Available from:

http://www.cebm.net/mod_product/design/files/CEBM-Levelsof-Evidence-2.1.pdf

12. Moraes M, Da Silva L, Faliú B, Sosa C. Técnica de alimentación a pecho y aparición de trauma del pezón previo al alta hospitalaria. Arch. Pediatr. Urug. [Internet].

2011 [cited 2012 jul 29] ; 82(1):10-17. Available from:

http://www.scielo.edu.uy/pdf/adp/v82n1/v82n1a03.pdf

13. Oliveira LD, Giugliani ER, Do Espírito Santo LC, Cavalheiro M, França T, Weigert EML, et al. Effect of Intervention to Improve Breastfeeding Technique on the Frequency of Exclusive Breastfeeding and Lactation-Related Problems. J Hum Lact. 2006 [cited 2012 ago 02]; 22(3):315-21.

14. Weigert EML, Giuglian ERJ, França MCT, De Oliveira LD, Bonilha A, Do Espírito Santo LC et al . Influência da técnica de amamentação nas freqüências de aleitamento materno exclusivo e lesões mamilares no primeiro mês de lactação. J. Pediatr. (Rio J.) [Internet]. 2005 [cited 2012 jul 29] ; 81(4): 310-316. Available from: http://www.scielo.br/pdf/jped/v81n4/v81n4a09.pdf 15. Melli MS, Rashidi MR, Nokhoodchi A, Tagavi S, Farzadi L, Sadaghat K, Tahmasebi Z, Sheshvan MK. A randomized trial of peppermint gel, lanolin ointment, and placebo gel to prevent nipple crack in primiparous breastfeeding women. Med Sci Monit. [Internet]. 2007 [cited 2012 jul 29];13(9):406-411.

Available from:

http://www.aromamd.net/edu_cracked_nipples.pdf

16. Centuori S, Burmaz T, Ronfani L, Fragiacomo M, et al. Nipple Care, Sore Nipples, and Breastfeeding: A Randomized Trial. J. Human Lact. 1999 [cited 2012 ago 02]; 15(2):125-130.

17. Newton N. Nipple pain and nipple damage; problems in the management of breast feeding. J Pediatr. 1952 [cited 2012 ago 02];41(4):411-23.

18. Filho MDF, Neto PNTG, Martins, MCC. Avaliação dos problemas relacionados ao aleitamento materno a partir do olhar da enfermagem. Cogitare Enferm. [Internet]. 2011 [cited 2012 jul 15];16(1):70-5. Available from: http://ojs.c3sl.ufpr.br/ojs2/index.php/cogitare/article/view/211 $14 / 13940$

19. Vieira F, Tonhá ACM, Martins DMC, Ferraresi MF, Bachion MM. Diagnósticos de enfermagem relacionados à amamentação no puerpério imediato. Rev Rene. [Internet]. 2011 [cited 2012 jul 28];12(3):462-70. Available from: http://www.revistarene.ufc.br/vol12n3 html site/a03v12n3.ht $\underline{\mathrm{m}}$

20. Coca KP, Abrão ACFV. Avaliação do efeito da lanolina na cicatrização dos traumas mamilares. Acta Paul Enferm. [Internet]. 2008 [cited 2012 jul 15];21(1):11-6. Available from: http://www.scielo.br/pdf/ape/v21n1/pt 01.pdf

21. Biancuzzo M. Sore nipples: prevention and problem solving. Herndon, USA: WMC Worldwide; 2000.

22. Silva PP, Silveira RB, Mascarenhas MLW, Silva MB, Kaufmann CC, Albernaz EP. A percepção das mães sobre o apoio paterno: influência na duração do aleitamento materno. Rev. Paul. Pediatr. [Internet]. 2012 [cited 2012 jul 15];30(3):306-313. Available from: http://www.scielo.br/pdf/rpp/v30n3/02.pdf 23. Piazzalunga CRC, Lamounier JA. A paternidade e sua influência no aleitamento materno. Pediatria (São Paulo). [Internet] 2009 [cited 2012 jul 15];31(1):49-57. Available from: http://www.pediatriasaopaulo.usp.br/upload/pdf/1290.pdf
24. Susin LR, Giugliani ER. Inclusion of Fathers in an Intervention to Promote Breastfeeding: Impact on Breastfeeding Rates. J Hum Lact. [Internet]. 2008 [cited 2012 jul 15]; 24(4):386-92. Available from:

http://repositorio.furg.br:8080/jspui/bitstream/1/2667/1/Inclusi on\%20of\%20Fathers\%20in\%20an\%20Intervention\%20to\%20Pr omote $\% 20$ Breastfeeding\%20-

\%20Impact\%20on\%20Breastfeeding\%20Rates.pdf

Artigo recebido em 27/02/2013.

Aprovado para publicação em 29/05/2013.

Artigo publicado em 30/09/2013. 